\title{
Optimization of Target Q-Coverage Problem for QoS Requirement in Wireless Sensor Networks
}

\author{
Recep ÖZDAĞ \\ Department of Computer Engineering, Faculty of Engineering, YuzuncuYilUniversity, Van, Turkey. \\ Corresponding author. Tel.: +904322251701; email: rozdag@yyu.edu.tr \\ Manuscript submitted April 15, 2017; accepted July 20, 2017. \\ doi: 10.17706/jcp.13.4.480-489
}

\begin{abstract}
In the recent times, Quality of Service (QoS) terminology has been frequently used for Wireless Sensor Networks (WSNs) because of the fact that the targets which are covered by a different number of sensor nodes have different detection quality. QoS of the WSNs is determined depending upon the number of sensors that cover each target, sampling fraction etc. factors. Therefore, the optimization of the target Qcoverage problem is an important issue as an effective way that increases the lifetime of the network in continuous monitoring of the remote physical targets with different QoS restrictions. In this study, targets will be ensured to be covered by different detection quality by optimizing the Q-coverage problem which significantly plays a role in determining the coverage quality requirement of each target point in the related area. For this purpose, a new approach has been proposed based on Electromagnetism-Like (EM) algorithm, which is meta-heuristic. As a result of the comparison of the proposed algorithm with Artificial Bee Colony (ABC) and Particle Swarm Optimization (PSO) algorithms, it was determined by the simulations made that the approach proposed in providing the Q-coverage requirements which were defined for all targets was an effective solution in increasing the lifetime of the network by decreasing the energy consumptions of the sensor nodes.
\end{abstract}

Key words: Network lifetime, Q-coverage, quality of service, target coverage problem.

\section{Introduction}

WSNs has attracted the attention of many researchers in the recent times due to their capability of processing and evaluating the data that are collected from the environment by being transmitted to base station through nodes for media monitoring. While WSNs has much more areas of utilization in military applications, they are also used in many different areas such as agricultural monitoring, earthquake monitoring, vehicle tracking and healthcare applications [1]. WSNs consist of sensor nodes with a limited amount of energy. Recharging of the batteries of the sensor nodes is not possible due to their limited battery sizes. Therefore, many researchers have proposed to reduce the energy consumptions to increase the lifetime of the sensor nodes as far as possible [2]-[4].

Unnecessary monitoring of all areas by the sensor nodes and extra battery power consumption of the nodes are prevented as a result of monitoring only a certain number of targets instead of monitoring all areas due to the large size of the areas where WSNs are applied and the limited amount of energy of the nodes.

The distribution of the nodes that make up the WSNs can be performed in a random or deterministic way. Random distribution is suitable for the applications in cases where the details of the area to be monitored 
are not known or it is not possible to reach the area. Monitoring of the battlefields can be given as an example for the random distribution of the sensor nodes [5]. Deterministic distribution is the distribution of the nodes by being close to certain targets to be monitored in cases where the details of the region are known previously. The coverage of the targets is blocked with more nodes than necessary by providing the coverage requirements of the targets with this distribution. Thus, the lifetime of the network is increased by decreasing the energy consumptions of the nodes. However, it is necessary to distribute more nodes than necessary in order to monitor the related area when random distribution is performed. The energy consumption of the nodes will increase because of the fact that this excess distribution will cause unnecessary coverage of some targets. Therefore, deterministic distribution [6] is usually preferred while performing the distribution of the sensor nodes in the related area.

Coverage problems can be classified as area coverage and target coverage [5].Although area coverage requires monitoring all of the related areas, target coverage requires monitoring the specific target points which are critical in the related area. Target coverage is classified as $k$-coverage and $Q$-coverage. $k$-coverage [7]means that $T_{j}$ target points which are in the range of $1 \leq j \leq n$ in $T=\left\{T_{1}, T_{2}, \ldots, T_{n}\right\}$ target point sets are covered by the least $k$ sensor node. $Q$-coverage [8] means that $T_{j}$ target points which are in the range of $1 \leq j \leq n$ in $Q=\left\{q_{1}, q_{2}, \ldots, q_{n}\right\}$ coverage vector set are covered by the least $q_{j}$ sensor nodes. While the targets in practical applications are required to be covered by the constant $k$ sensor nodes in order to ensure the coverage requirements of the targets, the targets in critical applications are required to be covered by a different number of sensor nodes which are defined in $Q$-coverage vector in order to ensure the coverage requirements of the targets. More realistic results are achieved by $Q$-coverage because of the fact that higher detection accuracy and fault tolerance are achieved by large number of sensor nodes for the targets which have different coverage requirements [9].

Many studies have been carried out in the literature for the optimization of the network's lifetime. The first basic version of the $Q$-coverage problem was formulated in [10]. In this study, $q_{k}=1$ was taken for all $k \in\{1,2, \ldots, m\}$ target points by assuming that all sensors had the same lifetimes. A central algorithm was proposed for $Q$-coverage problem in order to meet the QoS requirement by HESL (Heuristic with High Energy and Small Lifetime) which was developed heuristically in [11]. This heuristic algorithm uses the Greedy algorithm in order to create appropriate coverage by taking into account the priorities of the nodes according to remaining lifetime. In [12], a priority based heuristic algorithm was developed for the basic version of the $Q$-coverage. This algorithm assigns a priority value to each sensor node depending on the number of the targets covered and the remaining lifetime of the battery. Furthermore, Mini et al. [5] made the dynamic distributions of the sensor nodes in the manner that the lifetime of the network would be maximum by proposing a heuristic algorithm for the solution of the $k$-coverage and $Q$-coverage problems.

In this study, an attempt to reach the maximum network lifetime was made by mathematically calculating the upper limit of the lifetime of the network. For this purpose Target $Q$-Coverage algorithm based on Maximum Lifetime with EM algorithm, which is meta-heuristic (TQCML-EM) was developed. Along with TQCML-EM which was proposed as a new approach, dynamic distributions were performed by minimizing the energy consumptions of the sensor nodes in a way to meet the $Q$-coverage requirements of the WSNs. In addition, the simulation results which were calculated with the proposed method were compared with the $\mathrm{ABC}$ and PSO algorithms in the literature, and the effectiveness of the TQCML-EM in the solution of the $Q$ coverage problem was measured.

In this study, in Section 2, the target $Q$-coverage problem was defined as one of the factors that provide the QoS requirement of the WSNs. TQCML-EM which was developed for the solution of this problem is presented in Section 3. The simulation results calculated were discussed by comparing with ABC, PSO and 
random distribution in Section 4; and the final results obtained from this study are presented in Section 5.

\section{Problem Definition}

It is the ability to maximize the lifetime of the network through the fact that the $T_{j}$ target points which are in the range of $1 \leq j \leq n$ are covered by the least $q_{j}$ sensor nodes so that the QoS requirement can be met by defining the $T=\left\{T_{1}, T_{2}, \ldots, T_{n}\right\}$ target set consisting of $n$ pieces of points, $S=\left\{S_{1}, S_{2}, \ldots, S_{m}\right\}$ sensor set consisting of $m$ pieces of mobile node and $Q=\left\{q_{1}, q_{2}, \ldots, q_{n}\right\}$ coverage vector in the relevant area.

Definition2.1. Q-coverage Requirement.

The target coverage is performed based on three basic version of the $Q$-coverage problem [9].

- Basic Q-coverage Problem: It is the first basic version that assumes that the base station is positioned into the communication range of each sensor. The base station has a role of controlling the sensors as well as collecting the data detected by the sensor nodes.

- Q-coverage Problem with Variable Energy Consumption: It is the second version that assumes that the energy consumption of a sensor is dependent on the number of the target covered by that node.

- Q-coverage Problem with base station connectivity restrictions: As the third version of the problem, each sensor has a communication range in the manner a sensor node can make a direct (non-switched) contact with another sensor (or base station) which is positioned in its communication range. In addition, it is also possible that active sensor nodes can make an indirect (switched) contact with the base station due to the multihop transmission among themselves.

Definition2.2. Upper Limit of the Network Lifetime.

Assume that the nodes are distributed randomly in order for $m$ node in the $S$ sensor set to cover the $n$ target in the $T$ targets set in the relevant area, each node has the initial $E_{0}$ battery power and the detection radius of the node is $S_{r}$. If $T_{j}$ target point, when it is $1 \leq i \leq m$, is positioned into the detection radius of $S_{i}$ node, that node covers the target point. The coverage matrix given in Equation (1) is generated to determine the $T_{j}$ target points covered by the $S_{i}$ node.

$$
M_{i j}=\left\{\begin{array}{l}
1, R\left(S_{i}, T_{j}\right) \leq S_{r} \\
0, \text { otherwise }
\end{array}\right.
$$

where Euclidean distance [6] between $S_{i}$ and $T_{j}$ is represented by $R$. The remaining lifetime of each node was calculated by formula

$$
b_{i}^{\prime}=\frac{b_{i}}{e_{i}}
$$

where the initial battery power of $S_{i}$ node was represented by $b_{i}$, and its energy consumption rate was represented by $e_{i}$. By performing the dynamic distributions of nodes according to the $Q$-coverage requirement, the upper limit of the lifetime of the network after the coverage of all targets is calculated by Equation (3) [5], and thus maximum achievable lifetime of the network is determined.

$$
U_{W S N}=\min _{j}\left[\frac{\sum_{i} M_{i j} * b_{i}^{\prime}}{q_{j}}\right], q_{j} \in Q
$$

After ensuring the $Q$-coverage requirement for all $T_{j}$ target points, the sum of the remaining lifetime of the $S_{i}$ sensor nodes that cover the $T_{j}$ target point in the $M_{i j}$ coverage matrix and the number of $q_{j}$ nodes that cover that target point determine the lifetime of the network in the $T_{j}$ target point. According to Equation 
(3), the upper limit of the achievable lifetime of the network is determined by detecting the minimum network lifetime which is calculated for all $T_{j}$ target points.

\section{Proposed Method}

In this study, an attempt to optimize the lifetime of the network by ensuring the coverage of the target points, the locations of which in the area are randomly determined by the meta-heuristic algorithm using mobile nodes, according to QoS requirements was made. For this purpose, meta-heuristic [13] EM algorithm [14] was used in the dynamic distribution of the nodes. In addition, TQCML-EM was developed as a new method in order to minimize the energy consumptions of the sensor nodes and thus to maximize the lifetime of the network by ensuring the $Q$-coverage requirement for the target points which were defined after dynamic distribution.

\subsection{EM Algorithm}

It is a meta-heuristic algorithm that simulates the repulsive and pulling movements of the charged particles in the electromagnetic field and runs as population-based by optimizing the non-linear problems [14]. It runs over the sample points set in the $n$-dimensional area in the solution space. Each sample point acts as a charged particle. The fitness function value of this point is used to determine the load of a certain point. This load basically determines the repulsive and pulling force of the points along the population [15]. According to the theory of electromagnetism, the resultant force of each particle is calculated by taking the sum of the forces applied to it by the other particles in the population, and this particle updates its current position for the optimum solution by moving in the resultant force direction [16].

EM algorithm, the general steps of which are presented in Algorithm 1[17], consists of four basic procedures. These are identification, local search, calculating the total force and location update[14].

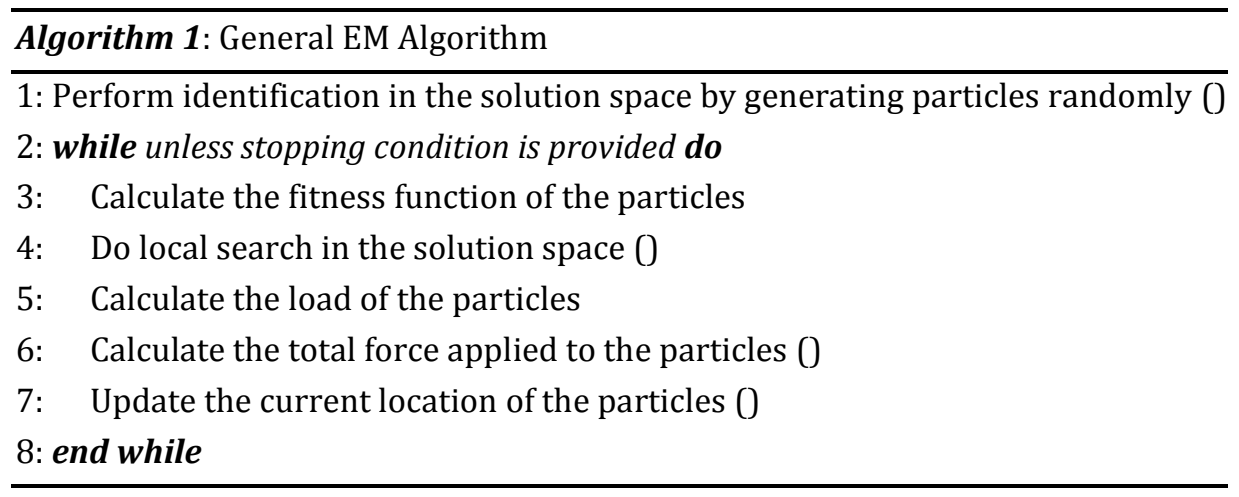

\subsection{Target $Q$-Coverage Algorithm Based on Maximum Lifetime}

By the TQCML-EM algorithm developed, it was aimed to make the dynamic distribution of the sensor nodes which were randomly distributed at the beginning by ensuring their less energy consumption as much as possible in order to optimize the QoS of the network. QoS means that $Q$-coverage requirement is ensured for all targets in order for all defined target points to be covered in a quality manner. Target $Q$ coverage requirement refers to the coverage of the $T_{j}$ target points by the least $q_{j}$ sensor node which is defined in variable number in the $Q$-coverage vector. Therefore, ensuring the $Q$-coverage requirement of the network can take place by the coverage of each target point with different numbers of nodes. TQCML-EM algorithm developed is shown in Fig. 1.

Both the initial distribution of the sensor nodes and determining the locations of the targets were performed randomly in each simulation in order to show that dynamic distribution of the nodes could be performed effectively by TQCML-EM. While performing dynamic distribution, initial $f_{x}^{i}$ value of each node 
was defined to be equal to the initial battery power of these nodes. Therefore, there will be a decrease in available battery power of each node that covers the target points as long as the $Q$-coverage requirement is ensured in the space, and thus it will be necessary to update $f_{x}^{i}$ values of these nodes. The dynamic distribution of the nodes is performed using the EM algorithm unless $Q$-coverage requirement cannot be ensured for all targets. In this process, if the battery power of the nodes that cover the targets is fully consumed, these nodes are put down from the network and are not included in the dynamic distribution. Because the node remains active in the space as long as it has energy and transmits the data it detects to the base station by scanning the target points.

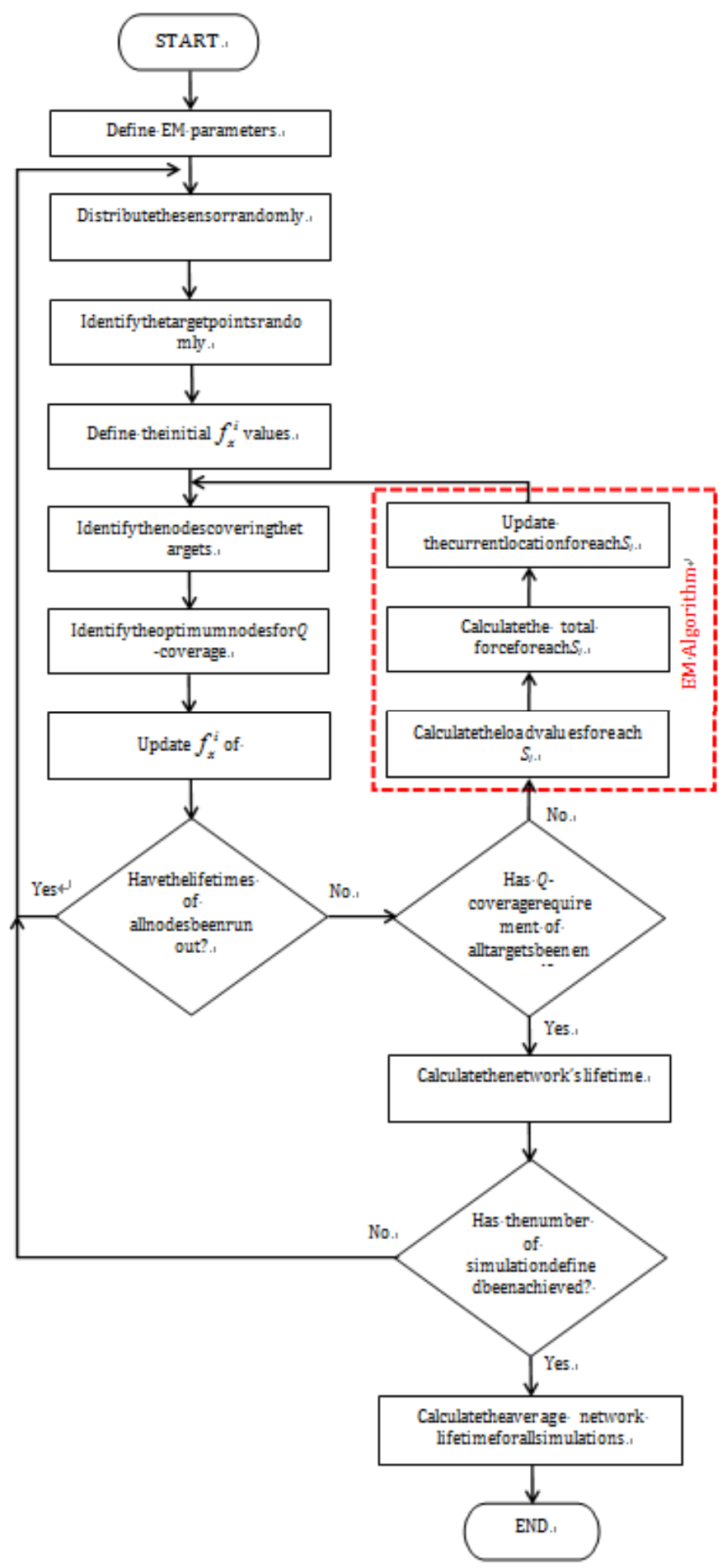

Fig. 1. TQCML-EM flowchart. 
Unless the number of the nodes that cover the $T_{j}$ target exceeds the $q_{j}$ number of nodes defined in the $Q$ coverage vector, $S_{i}$ node that covers the $T_{j}$ target is optimally determined, and its location in the space is fixed and acts as a static node in the next iterations. $Q$-coverage requirements of all targets are ensured rapidly due to the statically identification of the nodes, and the fact that the targets points are unnecessarily covered with excess nodes is prevented. Consequently, by this method proposed, the energy consumptions of the nodes are minimized and an attempt to reach the upper limit of the lifetime of the network is made by ensuring the rapid convergence of the nodes to targets.

\section{Experimental Results and Discussion}

In this study, the simulations of the proposed algorithm were performed using MATLAB R2014a program. The results obtained from the simulations which were performed to extend the lifetime of the network in order to optimize the QoS of the network by TQCML-EM were compared with the ABC and PSO algorithms studies in [5], and the performance of the proposed algorithm was measured.

\subsection{Dynamic Distribution by ABC and PSO Algorithms}

$\mathrm{ABC}$ and PSO algorithms, which were compared and studies in [5], were run by 5 Monte Carlo simulation. Detection radius distances of these sensors were defined as 75 meters in the dynamic distribution made by these algorithms. According to the graphics shown in Fig. 2, Fig. 3 and Fig. 4, ABC algorithm reached a relatively better network lifetime compared to PSO when the dynamic distribution of the nodes in the range of 100-250 was performed in the manner the $Q=[1-3], Q=[1-4]$ and $Q=[1-5]$ coverage requirement of 25 target points could be ensured.

\subsection{Random Distribution}

In the case that the nodes in the 100-250 range in the relevant space are distributed just by random distribution method by ensuring the $Q$-coverage requirement defined for each target point, the nodes will negatively affect the lifetime of the network by becoming passivated in a short time because the nodes will consume excessive amounts of energy. In the random distribution given in Fig. 2, it is necessary to define the detection range of the nodes as 35 meters and the number of the nodes as at least 200 in order to ensure the coverage requirement in the $Q=[1-3]$ range. In the case that $Q$-coverage range was increased to achieved $Q=[1-4]$ and $Q=[1-5]$, the defined $Q$-coverage requirement could be achieved by randomly distributing at least 250 nodes as it is shown in Fig. 3 and Fig. 4 because of the fact that the target points would need to be covered by more nodes. However, the lifetime of the network could not be calculated because coverage requirements could not be ensured when 100 and 150 nodes were distributed for $Q=[1-3]$ and 100,150 and 200 nodes were distributed for $Q=[1-4]$ and $Q=[1-5]$. Consequently, it is necessary to distribute excessive amounts of sensor nodes in order to ensure the $Q$-coverage requirements in the ranges which are defined by random distribution method. Therefore, increasing the number of the nodes in the space will lead to the unnecessary coverage of each target and consequently to the rapid reduction of the lifetime of the network by the increase of the energy consumption of the nodes.

\subsection{Dynamic Distribution by the Proposed Algorithm}

The maximum lifetime of the network was calculated using the parameters defined in Table 1 of the simulation of the TQCML-EM which was developed in order to minimize the energy consumptions of the nodes which were randomly distributed at the beginning in the relevant space. The detection radius distances of the nodes used in TQCML-EM were defined as 25 meters in order to show that the performance of the proposed algorithm was more effective by comparing with ABC and PSO algorithms studies in [5]. 20 Monte Carlo simulation was run in order to ensure the coverage requirements of the 25 target points, the 
locations of which in each simulation was randomly defined, in the relevant space which was defined as 250 $\mathrm{m}^{2}$ by the node distributed in the 100 and 250 range to be $Q=[1-3], Q=[1-4], Q=[1-5]$. Furthermore, the initial energy of each node was defined as 100 unit, and the energy consumption rate of these nodes were defined as 1 unit.

Table 1. Simulation Parameters for TQCML-EM

\begin{tabular}{l|l}
\hline \hline Relatedarea $(A)$ & $500 \times 500$ meter $\left(250 \mathrm{~m}^{2}\right)$ \\
\hline Number of nodes $(m)$ & $100-250$ range \\
\hline Number of targets $(T)$ & 25 piece \\
\hline Sensingradius of nodes $(r)$ & 25 meter \\
\hline Coverage range $(Q)$ & {$[1-3],[1-4],[1-5]$} \\
\hline Initiallifetime of thenode $i^{\text {th }}\left(b_{i}\right)$ & 100 unit \\
\hline Energyconsumption rate of thenode $i^{\text {th }}\left(e_{i}\right)$ & 1 unit \\
\hline Number of simulations $\left(N_{\text {sim }}\right)$ & 20 Monte Carlo \\
\hline \hline
\end{tabular}

According to the simulation results presented in Table 2, it was found that the average lifetime of the network was optimum according to the $\mathrm{ABC}$ and PSO algorithms by ensuring all coverage requirements in the $Q$-coverage set which was defined in the TQCML-EM developed. Therefore, maximum network lifetime was achieved in each simulation by minimizing the energy consumptions of the nodes by the algorithm developed.

Table 2. The Simulation Results of the Network Lifetimes Achieved by TQCML-EM, ABC[5] and PSO[5] Algorithms

\begin{tabular}{cccccccccc}
\hline \hline \multirow{2}{*}{$m$} & \multicolumn{3}{c}{$Q=[1-3]$} & \multicolumn{3}{c}{$Q=[1-4]$} & \multicolumn{3}{c}{$Q=[1-5]$} \\
\cline { 2 - 10 } & $\begin{array}{c}\text { TQCML- } \\
\text { EM }\end{array}$ & ABC & PSO & $\begin{array}{c}\text { TQCML- } \\
\text { EM }\end{array}$ & ABC & PSO & $\begin{array}{c}\text { TQCML- } \\
\text { EM }\end{array}$ & ABC & PSO \\
\hline 100 & 1242.50 & 253 & 247.6 & 816.25 & 244.6 & 209.56 & 420.42 & 185 & 179.2 \\
\hline 150 & 1690.00 & 406.4 & 406.4 & 1518.75 & 363.2 & 361.64 & 1201.25 & 280 & 279.32 \\
\hline 200 & 2083.75 & 466.2 & 466.2 & 1853.13 & 418.2 & 414.64 & 1608.75 & 360 & 358.8 \\
\hline 250 & 2173.75 & 573 & 569.04 & 2057.50 & 543.2 & 512.24 & 1917.50 & 440 & 435.8 \\
\hline \hline
\end{tabular}

In order to prove that the $Q$-coverage requirements of the network could be ensured effectively by TQCML-EM, simulations were performed by defining the detection radius distances of the nodes by twothirds of the ABC and PSO algorithms and as less than 10 meters according to the random distribution. According to the results represented graphically in Fig. 2, Fig. 3 and Fig. 4, the average network lifetime achieved was optimum according to the compared algorithms despite the fact that the detection radius distances of the nodes were less defined as 25 meters in the proposed algorithm.

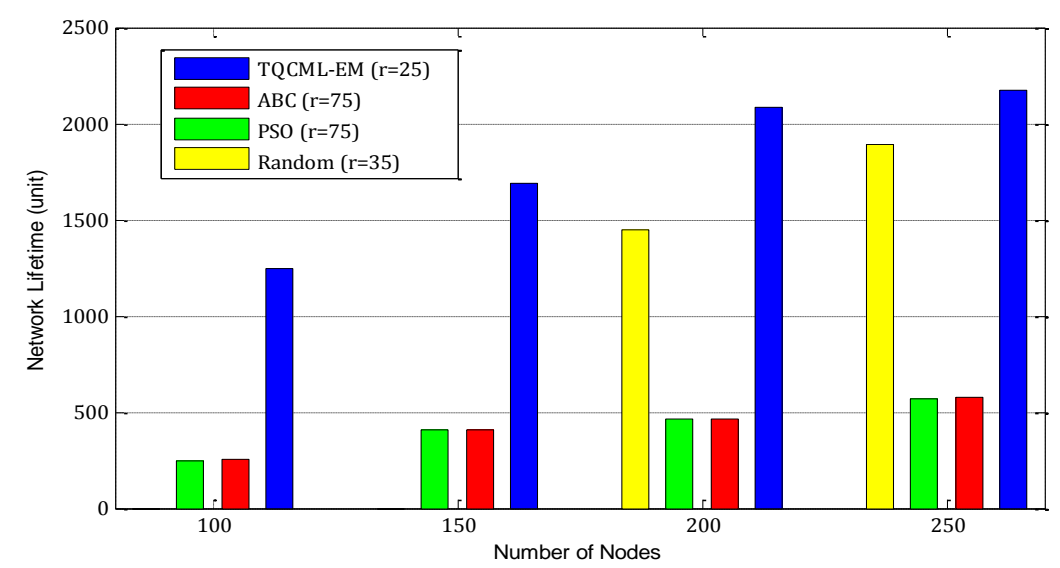

Fig. 2. Comparison of the network lifetimes for the coverage set in the range of $Q=[1-3]$. 


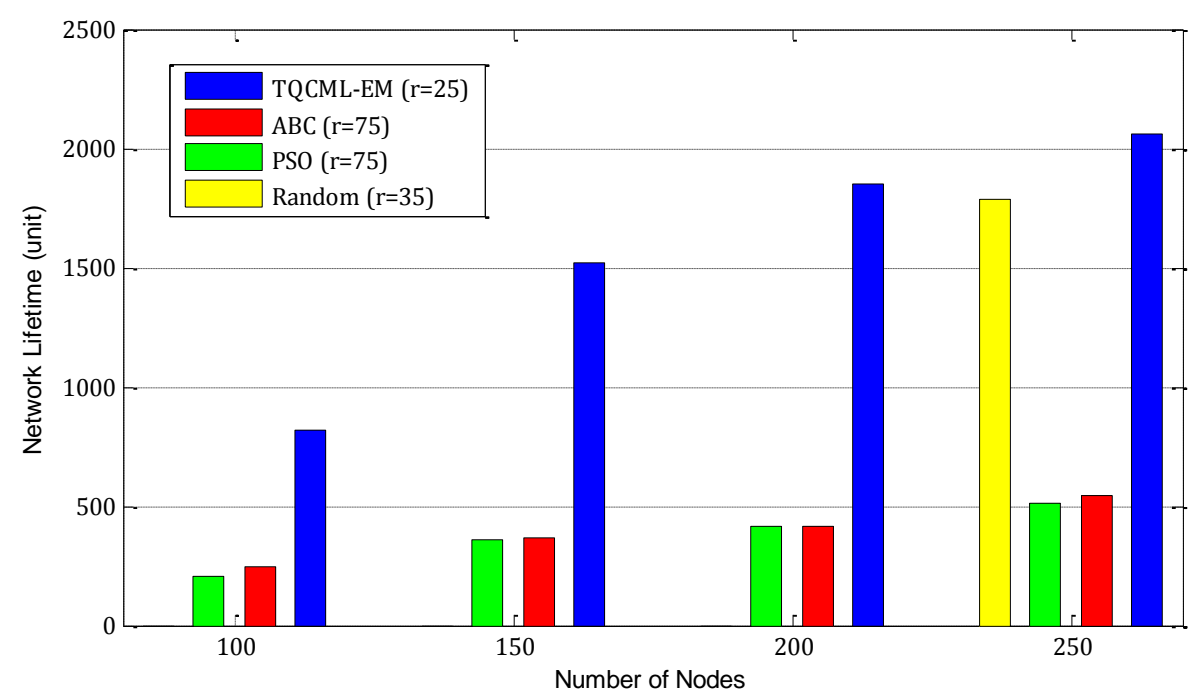

Fig. 3. Comparison of the network lifetimes for the coverage set in the range of $Q=[1-4]$.

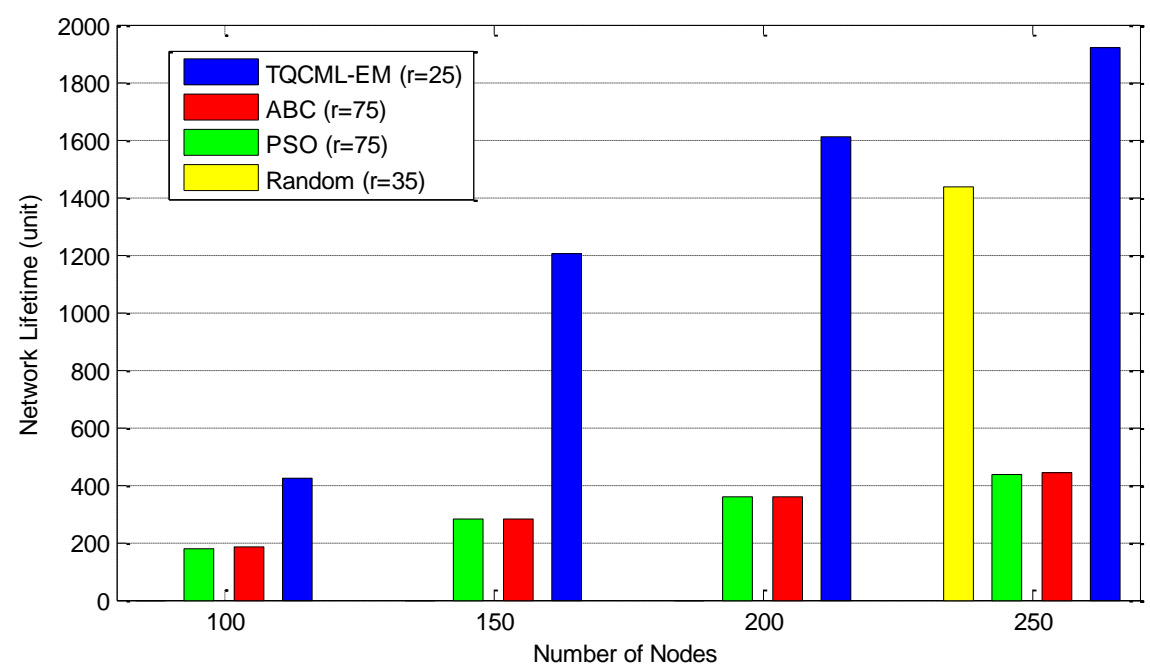

Fig. 4. Comparison of the network lifetimes for the coverage set in the range of $Q=[1-5]$.

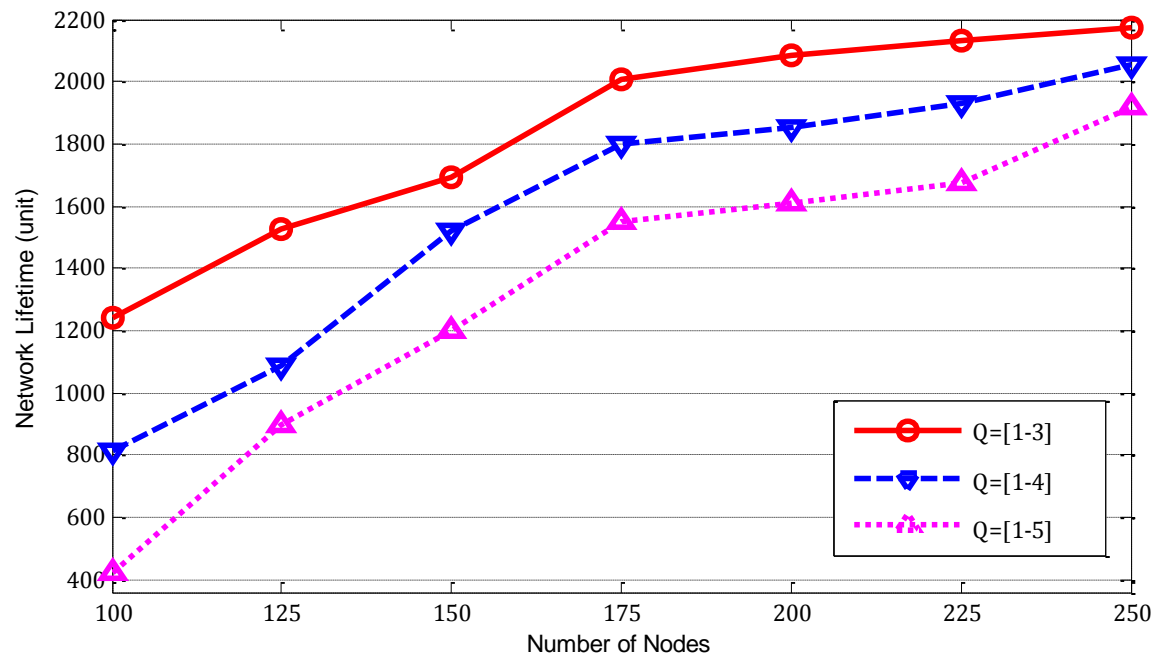

Fig. 5. The average network lifetimes achieved according to Q-coverage ranges by TQCML-EM.

The change chart of the average network lifetimes which were achieved by the $Q=[1-3], Q=[1-4]$ and $\mathrm{Q}=[1-5]$ coverage ranges defined by TQCML-EM for 25 target points in the area is presented in Fig. 5. 
According to this chart, the coverage requirements of these targets can be achieved by running more iterations in each simulation because of the fact that the number of nodes covering these targets will increase as the coverage requirement that should be ensured for target points increases. Therefore, increase in the number of iterations causes the reduction of the lifetime of the network by increasing the energy consumptions of the nodes. In addition, the energy consumptions of the nodes will decrease and thus the increase of the average network lifetime will be ensured because of the fact that the coverage requirements of the target points will be met rapidly as the number of the nodes in the area increases for each $Q$-coverage range defined.

\section{Conclusion}

In this study, a new method that maximizes the lifetime of the network and performs the dynamic distribution of the nodes based on EM algorithm in this process was developed by ensuring the $Q$-coverage requirements of the nodes which were randomly distributed at the beginning in the defined ranges in order to optimize the QoS of the network. The calculated simulation results were compared with meta-heuristic ABC, PSO algorithms and random distribution method in order to prove that $Q$-coverage requirements of the targets could be ensured effectively by the TQCML-EM developed. According to the simulation results, the average network lifetime achieved was found to be lower despite the fact that the node radius distances in the ABC and PSO algorithms were defined two times more than the TQCML-EM. Besides, it was found that $Q$-coverage requirements could be achieved in the case that the nodes are only distributed intensively in the random distribution method, but nodes become passivated rapidly by consuming excessive amount of energy in this situation.

Because of the fact the purpose of the $Q$-coverage, which is one of the factors that provide the network's QoS requirements, is to ensure the continuity of the coverage of the target points in critical applications by ensuring the coverage of each target point by different numbers of sensor nodes, it was found by the results of the comparison made with ABC and PSO that this purpose was optimally ensured with the TQCML-EM developed. Therefore, in this study, meta-heuristic TQCLML-EM was proposed as a new method that maximizes the average lifetime of the network by minimizing the energy consumptions of the nodes and ensuring the $Q$-coverage requirements of all target points, and this is believed to contribute to the studies in this field.

\section{References}

[1] Ding, Y. S., Lu, X. J., Hao, K. R., Li, L. F., \& Hu, Y. F. (2011). Target coverage optimisation of wireless sensor networks using a multi-objective immune coevolutionary algorithm. International Journal of Systems Science, 42(9), 1531-1541.

[1] Bahi, J. M., Makhoul, A., \& Mostefaoui, A. (2008). Hilbert mobile beacon for localisation and coverage in sensor networks. International Journal of Systems Science, 39(11), 1081-1094.

[2] Aneja, Y. P., Chandrasekaran, R., Li, X., \& Nair, K. P. K. (2010). A branch-and-cut algorithm for the strong minimum energy topology in wireless sensor networks. European Journal of Operational Research, 204(3), 604-612.

[3] Ma, L., Wang, Z., Niu, Y., Bo, Y., \& Guo, Z. (2011). Sliding mode control for a class of nonlinear discretetime networked systems with multiple stochastic communication delays. International Journal of Systems Science, 42(4), 661-672.

[4] Mini, S., Udgata, S. K., \& Sabat, S. L. (2014). Sensor deployment and scheduling for target coverage problem in wireless sensor networks. IEEE Sensors Journal, 14(3), 636-644.

[5] Özdă̆, R., \& Karcı, A. (2014). Elektromagnetizma-like algoritması ile kablosuz algılayıcı ağların 
olasılıksal dinamik dağıtımları. Proceedings of the 2nd International Symposium on Innovative Technologies in Engineering and Science (ISITES) (pp. 368-377).

[6] Özdağ, R. (2016). The solution of the $k$-coverage problem in wireless sensor networks. Proceedings of the 24th Signal Processing and Communications Applications Conference (SIU) (pp. 873-876).

[7] Li, D., Liu, H., Lu, X., Chen, W., \& Du, H. (2012). Target Q-coverage problem with bounded service delay in directional sensor networks. International Journal of Distributed Sensor Networks, 10.

[8] Singh, A., Rossi, A., \& Sevaux, M. (2013). Matheuristic approaches for $Q$-coverage problem versions in wireless sensor networks. Engineering Optimization, 45(5), 609-626.

[9] Gu, Y., Liu, H., \& Zhao, B. (2007). Target coverage with QoS requirements in wireless sensor networks. Proceedings of the 2007 International Conference on Intelligent Pervasive Computing (IPC) (pp. 35-38).

[10] Chaudhary, M., \& Pujari, A. K. (2009). Q-Coverage problem in wireless sensor networks. Proceedings of the 10th International Conference on Distributed Computing and Networking (ICDCN) (pp. 325-330).

[11] Mini, S., Udgata, S. K., \& Sabat, S. L. (2011). A heuristic to maximize network lifetime for target coverage problem in wireless sensor networks. Adhoc \& Sensor Wireless Networks, 13(3-4), 251-269.

[12] Canayaz, M., Karci, A. (2016). Cricket behaviour-based evolutionary computation technique in solving engineering optimization problems. Applied Intelligence, 44(2), 362-376.

[13] Birbil, S. I., \& Fang, S. C. (2003). An electromagnetism-like mechanism for global optimization. Journal of Global Optimization, 25(3), 263-282.

[14] Özdağ, R., \& Karcl, A. (2015). A novel routing protocol based on EM-L algorithm for energy efficiency of wireless sensor networks. Proceedings of the 23th Signal Processing and Communications Applications Conference (SIU) (pp. 871-874).

[15] Özdağ, R., \& Karcl, A. (2015). Sensor node deployment based on electromagnetism-like algorithm in mobile wireless sensor networks. International Journal of Distributed Sensor Networks, 15.

[16] Özdağ, R., \& Karcl, A. (2016). Probabilistic dynamic distribution of wireless sensor networks with improved distribution method based on electromagnetism-like algorithm. Measurement, 79, 66-76.

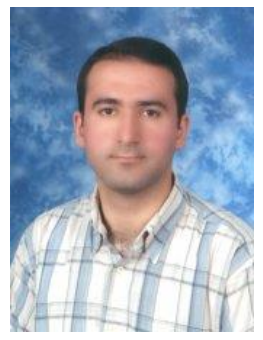

Recep Özdağ was born in Turkey in 1978. He received the B.S. degree in computer education from the Industrial Arts Education Faculty, Gazi University, Ankara, Turkey, in 2000. In 2007, he received the M.S. degree in electric and electronic engineering from Engineering Faculty, Yuzuncu Yil University, Van, Turkey. He received the Ph.D. degree in computer engineering from Engineering Faculty, Inonu University, Malayta, Turkey, in 2015.

He is an assistant professor of Engineering Faculty, Yuzuncu Yil University, Van, Turkey. Since 2010, he has been working as a network specialist in Computer Sciences Research and Application Center, Yuzuncu Yil University. His research fields are wireless sensor networks, ad-hoc sensor networks, meta-heuristic algorithms. 